\title{
A study on setting standards for near-infrared fluorescence-image guided surgery (NIRFGS) time lapse monitoring based on preoperative liver function assessment
}

\author{
Yunlong Li ${ }^{1 \#}$, Qi You ${ }^{1 \#, ~ Z i y a n g ~ W a n g ~}{ }^{1}$, Ying Cao ${ }^{1}$, Christopher J. Butch ${ }^{1}$, Nida El Islem Guissi ${ }^{1 \wedge}$, \\ Huiming Cai ${ }^{1,2}$, Yiqing Wang $^{1 \wedge}$, Qian Lu ${ }^{1,3}$ \\ ${ }^{1}$ Department of Biomedical Engineering, College of Engineering and Applied Sciences, State Key Laboratory of Analytical Chemistry for Life \\ Science, Nanjing University, Nanjing, China; ${ }^{2}$ Department of Research and Development Center, Nanjing Nuoyuan Medical Devices Co. Ltd., \\ Nanjing, China; ${ }^{3}$ Key Laboratory of Marine Drugs, Chinese Ministry of Education, School of Medicine and Pharmacy, Ocean University of China, \\ Qingdao, China \\ Contributions: (I) Conception and design: Y Wang; (II) Administrative support: H Cai; (III) Provision of study materials or patients: Y Wang; (IV) \\ Collection and assembly of data: Y Wang, Y Li, Q You; (V) Data analysis and interpretation: Y Wang, Y Li, Z Wang; (VI) Manuscript writing: All \\ authors; (VII) Final approval of manuscript: All authors. \\ "These authors contributed equally to this work. \\ Correspondence to: Huiming Cai, Master; Yiqing Wang, PhD; Qian Lu, PhD. Department of Biomedical Engineering, College of Engineering \\ and Applied Sciences, Nanjing University, No. 22, Hankou Road, Gulou District, Nanjing 210093, China. Email: chm9166@aliyun.com; \\ wangyiqing@nju.edu.cn; lq7080@ouc.edu.cn.
}

Background: This study aimed to explore the relationship between the fluorescence intensity of indocyanine green (ICG) in near-infrared fluorescence guided surgery (NIRFGS) and preoperative liver function indicators.

Methods: A total of 12 4T1 tumor-bearing mice were used for model establishment. Intraperitoneal injection (i.p.) of $20 \%$ carbon tetrachloride $\left(\mathrm{CCl}_{4}\right)$ corn oil solution $(50 \mu \mathrm{L})$ was given to mice in the liver injury model group, 24 hours after injection, the model was established, while the control group received $0 \% \mathrm{CCl}_{4}$ corn oil solution $(50 \mu \mathrm{L})(\mathrm{n}=6$ for each group). Additionally, doses of $8 \mathrm{mg} / \mathrm{kg}$ and $1 \mathrm{mg} / \mathrm{kg}$ of free ICG were injected intravenously (i.v.) ( $\mathrm{n}=3$ in each group). Fluorescence was imaged in vivo using an NIR fluorescence imaging system at different time points $(1,2,4,8,12,24,48$, and $72 \mathrm{~h})$ after injection.

Results: The absolute fluorescence intensity of mice in the liver injury model group was stronger than that in the control group. Mice in the liver injury model group had the same clearance rate of ICG from the tumor as normal mice. However, the background clearance rate was slower than that of normal mice, which prolonged the optimal tumor to background ratio (TBR) time. Correlation analysis was also used to determine which preoperative liver function parameters were most correlated with hepatic ICG clearance.

Conclusions: Liver injury does not significantly affect the maximum TBR, but prolongs the optimal TBR time, and at the same time, a wider and more stable surgical window will appear. This study showed that a prolonged surgical start time is feasible according to preoperative liver function testing using NIR fluorescence imaging technology.

Keywords: Near-infrared fluorescence guided surgery (NIRFGS); individual administration; liver function; tumor to background ratio (TBR)

Submitted Dec 07, 2021. Accepted for publication Jan 21, 2022.

doi: 10.21037/atm-21-6975

View this article at: https://dx.doi.org/10.21037/atm-21-6975

^ ORCID: Christopher J. Butch, 0000-0003-3112-0470; Nida El Islem Guissi, 0000-0003-4425-7580; Yiqing Wang, 0000-0002-5626-8589. 


\section{Introduction}

Cancer is a major threat to human health, with an estimated 19.3 million new cancer cases and nearly 10 million cancer deaths worldwide in 2020 (1). At present, surgical removal remains a mainstay for the cure and control of most solid cancers (2). The survival of patients with tumors depends on the ability to perform a complete resection. Surgeons use ultrasound, computed tomography (CT), magnetic resonance imaging (MRI), and other means to facilitate early detection, improve diagnostic accuracy and staging, and improve preoperative planning (3). In the common practice of surgical resection, surgeons rely on their senses of sight and touch to delineate the margin between the tumor and surrounding normal tissues. Approximately $40 \%$ of surgeries show signs of residual tumor cells in the surgical cavity after resection due to surgeons' subjective impressions (4,5). Thus, more sophisticated techniques are desired for attaining more precise determinations of tumor margins to diminish recurrence rates after surgery.

Near-infrared fluorescence guided surgery (NIRFGS) is one of the most promising techniques for precise surgical navigation of tumor excision, which offers a better distinction between cancerous and normal tissues compared to surgeries relying on the senses of sight and touch of a surgeon. The most dominant light absorbing substances in tissues are hemoglobin (including oxyhemoglobin and reduced hemoglobin) and water molecules. The absorption coefficient of hemoglobin and water molecules in a segment of NIR region of 650-900 nm (now known as NIR I) is 1-2 orders of magnitude lower than that in other regions (6). The low absorption coefficient also means that NIR light will not destroy the structure of biomolecules like ultraviolet (uv) light and does not harm normal tissues. NIR fluorescence can effectively avoid the auto-fluorescence of biological tissues, thereby improving the TBR, with the advantages of deep penetration depth and high safety (7-9). NIRFGS mainly uses indocyanine green (ICG) as the contrast agent, the only NIR fluorescent tracer approved by both the Chinese and US Food and Drug Administrations (CFDA and FDA) for clinical use $(10,11)$. The mechanism of NIR fluorescence-guided surgery is the Enhanced Permeability and Retention Effect (EPR) of tumors (12): ICG binds to proteins (mainly albumin) in plasma after i.v. into the human body to form nanostructures with a size of 4-6 nm. The new blood vessels in the tumor have holes that can be passed by nanoparticles because of incomplete structure, so the tumor has high permeability for nanostructured substances; and the lymphatic system in the tumor is scarce and lymphatic reflux dysfunction makes the nano-sized substances easily enter the tumor tissue and are not easily removed by lymphatic reflux, making the dye accumulation in the tumor higher than that in the normal tissue. Troyan et al. conducted a clinical trial of sentinel lymph node (SLN) tracing for breast cancer using the FLARE device in 2009 (13), and the first NIR fluorescence navigation surgery had very successful imaging results. With the development of the technology, the detection rate of SLNs can reach up to $95 \%$ (14). Subsequently, this technique has achieved outstanding results in a variety of tumor types, such as liver cancer $(15,16)$, lung cancer (17-19), and colorectal cancer (20). However, under the same ICG injection dose, the time of optimal TBR varies between different individuals (21-23). As the only metabolic organ of ICG, the liver's ability to metabolize ICG directly determines the trend of TBR $(21,24)$. Some researchers proposed that the preoperative period may be related to the degree of liver cirrhosis in patients (25), but there is no feasible NIR indicator dosing regimen to ensure that patients can obtain a good TBR according to preoperative liver function indicators. In this study, we artificially created different degrees of liver injury by injecting different concentrations of $\mathrm{CCl}_{4}$ into mice (Figure 1), and the objectives were: (I) to determine preoperative liver function indicators related to hepatic ICG metabolic capacity; (II) to predict the relationship between optimal TBR time and hepatic ICG metabolic capacity. This study provides us with a scientific basis and principles for the development of individualized dosing regimens for NIRFGS in the future, has the potential to solve key problems affecting the development and clinical application of NIRFGS, and promotes the progress of the precise surgical treatment of tumors. We present the following article in accordance with the ARRIVE reporting checklist (available at https://atm. amegroups.com/article/view/10.21037/atm-21-6975/rc).

\section{Methods}

\section{Cell cultures}

The human breast cancer cell line 4T1 was purchased from Jiangsu KeyGEN BioTECH Corp., Ltd. (Nanjing, China). Cells were cultured according to the vendors' recommendations. The choice of tumor cells was not restricted, 4T1 was chosen because it is a murine cell with high invasiveness and metastasis, making it easier to 

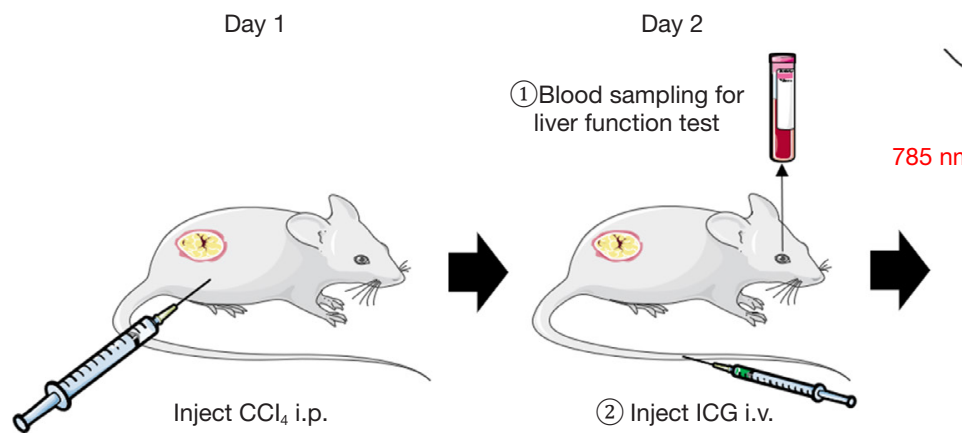

Figure 1 Scheme of the live imaging and workflow. i.p., intraperitoneal injection; i.v., Intravenous injection.

establish a tumor model.

\section{Mouse model}

Experiments were reviewed and approved by the Animal Ethical and Welfare Committee (AEWC) of Nanjing University (Approval No. IACUC-2105007), in compliance with Institutional Committee of Nanjing University guidelines for the care and use of animals. Female mice (6 weeks old, 18 \pm 2 g, Jiangsu Jicuaiyaokang Biotechnology Co., Ltd., China) received subcutaneous injection of 4T1 cells $\left(5 \times 10^{6}\right)$ in the right hind limb. For 4T1, the prevalence was higher and more general in female mice. Tumor volume was calculated as $\pi / 6 \times$ length $\times(\text { width })^{2}$, and mice with tumors of about $500 \mathrm{~mm}^{3}$ in size were selected for in vivo studies.

\section{Establishment of the liver injury model induced by CCl4 in mice}

Animals in the injury group were given an intraperitoneal injection of $20 \% \mathrm{CCl}_{4}$ corn oil solution $(0.5 \mu \mathrm{L} / \mathrm{g}, \mathrm{n}=3$ for each group). The control group received a subcutaneous injection of corn oil $(50 \mu \mathrm{L})$. The feeding conditions of the above animals were the same, with free access to food and water.

\section{Detection of liver function indicators}

Mice were fasted for $24 \mathrm{~h}$ the day before the end of the experiment, and blood samples $(100-200 \mu \mathrm{L})$ were taken from the orbit after anesthesia to measure 8 liver function indicators: alanine aminotransferase (ALT), aspartate aminotransferase (AST), direct bilirubin (DBIL), total bilirubin (TBIL), albumin (ALB), alkaline phosphatase (ALP), $\gamma$-glutamyltransferase $(\gamma-G T)$, and total bile acid (TBA). Blood samples were collected from mice and centrifuged at 4,000 rpm for 4 min twice to obtain serum. The above 8 liver function indicators were measured. The specific steps were performed according to the instructions of the kit.

\section{ICG injection and NIR fuorescence imaging}

ICG was purchased from Dandong Yichuang Pharmaceutical Co., Ltd. (Dandong, China). Doses of 8 and $1 \mathrm{mg} / \mathrm{kg}$ free ICG were injected via the tail vein $(\mathrm{n}=9$ in each group). Mice were anesthetized with intraperitoneal injection of $2 \%$ sodium pentobarbital, and fluorescence was imaged in vivo using an FLI-10B fluorescence navigation system (Nanjing Nuoyuan Medical Devices Co., Ltd., Nanjing, China) at different time points $(1,2,4,8,12$, 24, 48, and $72 \mathrm{~h}$ ) after injection (Figure S1). Imaging was performed $72 \mathrm{~h}$ post-injection to determine the fluorescence in the heart, liver, spleen, lung, kidney, stomach, small intestine, large intestine, and tumor tissue. After imaging ex vivo, frozen and hematoxylin and eosin-stained 4- $\mu \mathrm{m}-$ thick tissue sections of the tumor and liver were evaluated histopathologically.

\section{Statistical analysis}

\section{Calculation of TBR}

Five points were selected on both the tumor tissue and normal tissue $2 \mathrm{~cm}$ from the tumor boundary for the fluorescence images. TBR was calculated as follows: $\mathrm{TBR}=$ average fluorescence intensity of tumor/average fluorescence intensity of normal tissue. 


\section{Correlation analysis}

Since the fluorescence intensity of the tumor was continuously decaying after $1 \mathrm{~h}$, the fluorescence intensity of the tumor and background tissue at $1 \mathrm{~h}$ was defined as $\mathrm{I}_{0}\left(\mathrm{I}_{0-\mathrm{t}}, \mathrm{I}_{0-\mathrm{b}}\right)$. The correlation relationships between $\mathrm{I}_{0}$, halflife, and the 8 liver function indicators were assumed to be linear due to the small sample size. When multiple factors might have had a simultaneous impact on measurements (e.g., multiple liver function indicators), we used multiple linear regression to construct a linear correlation between 2 variables.

\section{Results}

\section{In vivo and ex vivo fluorescence imaging}

In this study, ICG injection doses were 8 and $1 \mathrm{mg} / \mathrm{kg}$ ( $\mathrm{n}=6$ in each group). At each dose, $20 \% \mathrm{CCl}_{4}$ was intraperitoneally injected to establish the liver injury model, as well as $0 \%$ $\mathrm{CCl}_{4}$ in the control group ( $\mathrm{n}=3$ in each group). The results are shown in Figure 2.

As shown in Figure $2 A$, intravenous injection of $8 \mathrm{mg} / \mathrm{kg}$ of ICG and intraperitoneal injection of $20 \% \mathrm{CCl}_{4}$ in corn oil solution resulted in a much higher fluorescence intensity of the tumor than the surrounding tissues, with the best tumor imaging effect and the clearest tumor boundary (the optimal TBR $=3.496 \pm 0.259$ ).

Intravenous injection of $8 \mathrm{mg} / \mathrm{kg}$ of ICG and intraperitoneal injection of $0 \% \mathrm{CCl}_{4}$ in corn oil (Figure $2 \mathrm{~B}$ ) showed higher contrast, relatively better tumor imaging, and relatively clear tumor boundary (the optimal TBR $=3.807 \pm 0.259$ ).

Intravenous injection of $1 \mathrm{mg} / \mathrm{kg}$ of ICG and intraperitoneal injection of $20 \% \mathrm{CCl}_{4}$ in corn oil (Figure 2C) resulted in poor tumor imaging and weak fluorescence intensity (the optimal TBR $=1.521 \pm 0.270$ ).

Intravenous injection of $1 \mathrm{mg} / \mathrm{kg}$ of ICG and intraperitoneal injection of $0 \% \mathrm{CCl}_{4}$ in corn oil (Figure $2 \mathrm{D}$ ) resulted in the lowest tumor imaging effect, weak fluorescence, and invisible tumors (the optimal TBR $=1.629 \pm 0.308$ ).

Therefore, the analysis focused on the $8 \mathrm{mg} / \mathrm{kg}$ ICG injection dose group in the subsequent experiments. Fluorescence intensity data and images were acquired using an FLI-10B imaging system (Figure 2).

\section{Optimal tumor visualization time for ICG NIRFGS}

The effect of the ICG dose on tumor imaging was first assessed, and the TBR curve was plotted for the high- and low-dose groups ( $\left.8 \mathrm{mg} / \mathrm{kg}, 0 \% \mathrm{CCl}_{4} v s .1 \mathrm{mg} / \mathrm{kg}, 0 \% \mathrm{CCl}_{4}\right)$ (Figure $3 A$ ). It was found that the TBR 4-72 $\mathrm{h}$ after ICG injection at the dose of $8 \mathrm{mg} / \mathrm{kg}$ was greater than 2, with good clinical visibility. However, the TBR of the $1 \mathrm{mg} / \mathrm{kg}$ dose group was $<2$ at all time points. Therefore, the highdose group had a better imaging effect and better TBR from the perspective of the mean value of TBR and image visibility (Figure 3B). Thereafter, the analysis focused on the high-dose group. The TBR of the $20 \% \mathrm{CCl}_{4}$ model group still showed an increasing trend until $72 \mathrm{~h}$ after ICG injection (TBR $=3.496 \pm 0.259)$, while the TBR of the $0 \%$ $\mathrm{CCl}_{4}$ control group reached the peak value at $24 \mathrm{~h}$ after ICG injection (TBR $=3.807 \pm 0.259$ ), and then showed a decreasing trend. Considering the TBR and the width of the surgical window, it was found that although the TBR value of the high-dose liver injury model group was slightly lower than that of the control group within $72 \mathrm{~h}$ after ICG injection, it had a wider and more stable imaging window.

\section{Effect of liver injury on tumor and background fluorescence decay rate}

For the $8 \mathrm{mg} / \mathrm{kg}$ high-dose ICG injection group, there was no significant difference in the tumor fluorescence intensity decay rate and half-life between the $20 \%$ $\mathrm{CCl}_{4}$ liver injury model group and the control group (Figure $4 A, 4 B$ ). However, there was a significant difference in the background fluorescence intensity decay rate and half-life (Figure 4B,4C), and the background fluorescence decay rate of the $20 \% \mathrm{CCl}_{4}$ liver injury model group was slower than that of the control group.

Table 1 summarizes the absolute tumor and background fluorescence intensities of the liver injury model group and the control group at 8 time points after $8 \mathrm{mg} / \mathrm{kg}$ highdose ICG injection. It can be seen that the $\mathrm{I}_{0-\mathrm{T}}$ and $\mathrm{I}_{0-\mathrm{B}}$ of the liver injury model group were significantly higher than those of the control group. For ease of comparison, we normalized all absolute fluorescence intensities to visually confirm the conclusions of Figure 4.

\section{Correlation between liver function indicators and ICG metabolism}

The classical chemical liver injury model was established by intraperitoneal injection of $20 \% \mathrm{CCl}_{4}$ corn oil solution. It was found that for the high-dose injection group at $8 \mathrm{mg} / \mathrm{kg}$, there were significant differences in the concentrations of ALT, 


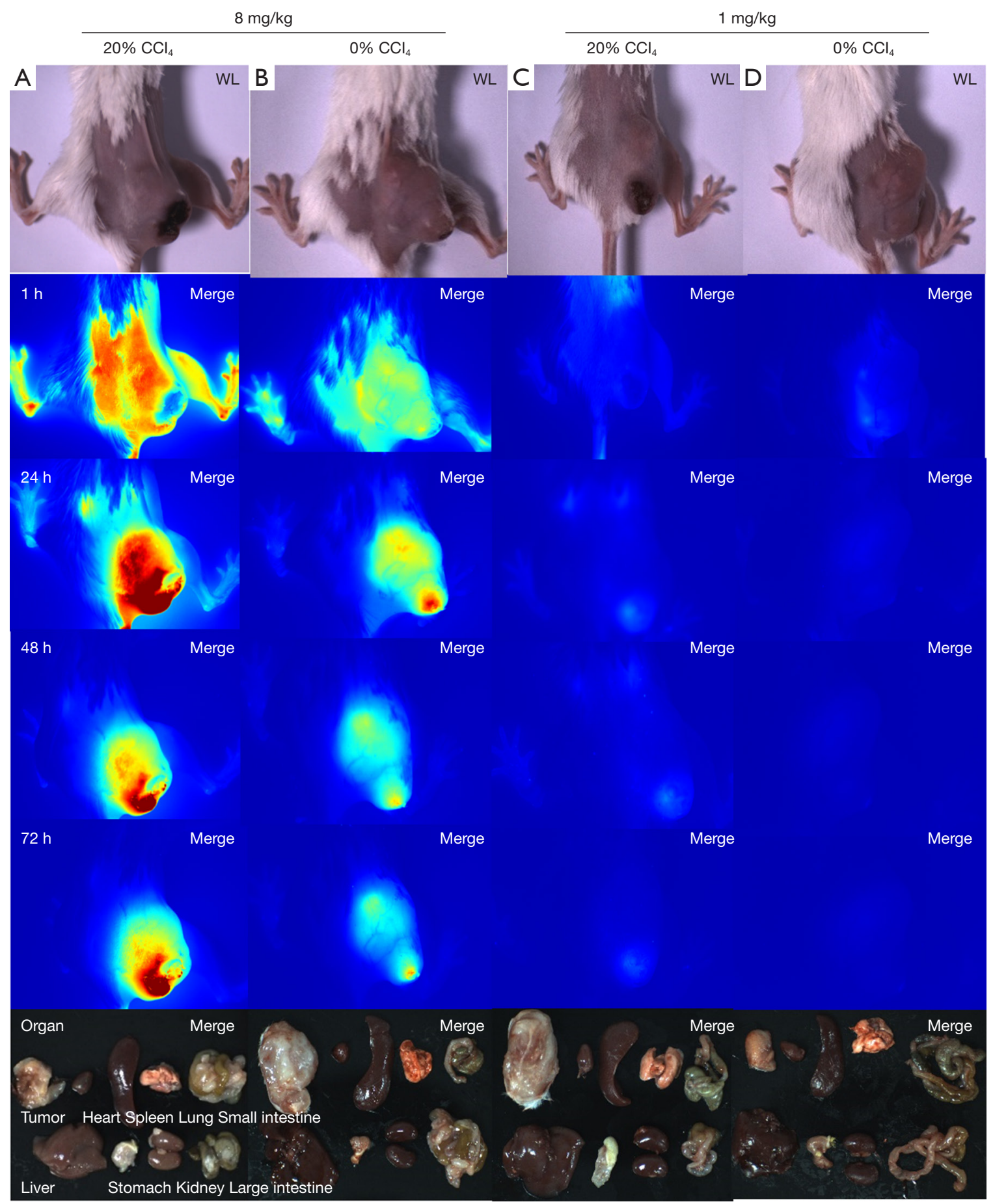

Figure 2 Tumor and organ images of mice in the imaging groups at different times. (A) Mice administered $8 \mathrm{mg} / \mathrm{kg}$ and $20 \% \mathrm{CCl}_{4}$ were imaged at 1, 24, 48, and $72 \mathrm{~h}$ after injection, white light images and merged images; (B) mice administered $8 \mathrm{mg} / \mathrm{kg}$ and $0 \% \mathrm{CCl}{ }_{4}$ were imaged after injection, white light images and merged images; (C) mice administered $1 \mathrm{mg} / \mathrm{kg}$ and $20 \% \mathrm{CCl}_{4}$ were imaged after injection, white light images and merged images; (D) mice administered $1 \mathrm{mg} / \mathrm{kg}$ and $0 \% \mathrm{CCl}_{4}$ were imaged after injection, white light images and merged images. 

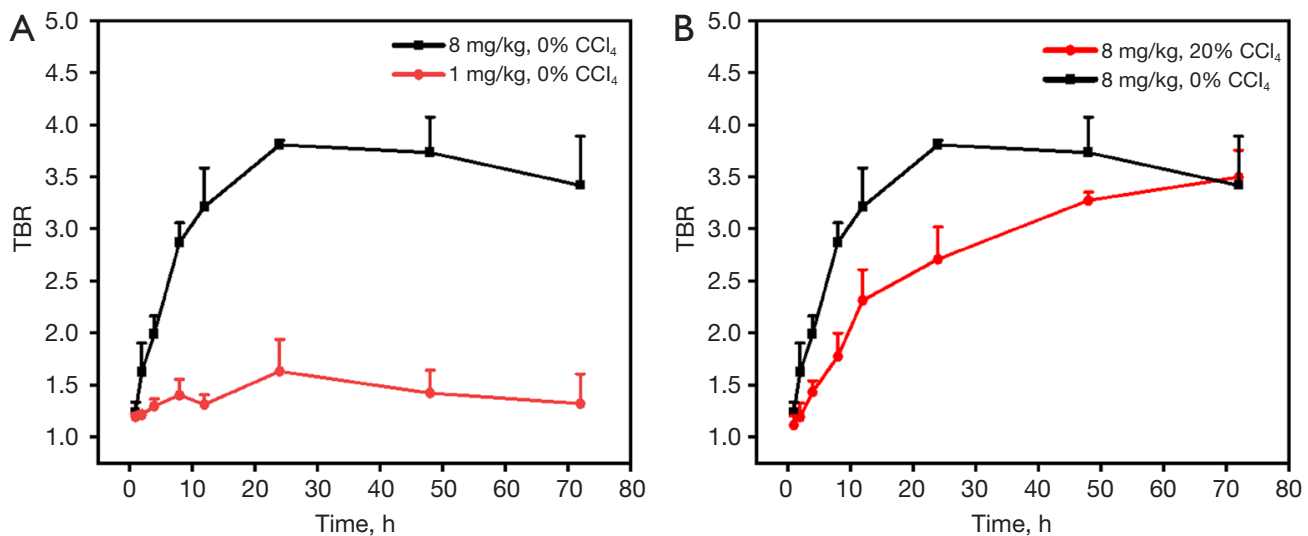

Figure 3 Comparison of TBR at different doses and degrees of liver injury. (A) TBR at different time points in groups of $8 \mathrm{mg} / \mathrm{kg}, 0 \% \mathrm{CCl}{ }_{4}$ and $1 \mathrm{mg} / \mathrm{kg}, 0 \% \mathrm{CCl}_{4}$. The former showed better TBR. (B) TBR at different time points in the $8 \mathrm{mg} / \mathrm{kg}, 20 \% \mathrm{CCl}_{4}$ and $8 \mathrm{mg} / \mathrm{kg}, 0 \% \mathrm{CCl}_{4}$ groups. The former had a wider clinical TBR window. TBR, tumor to background ratio.
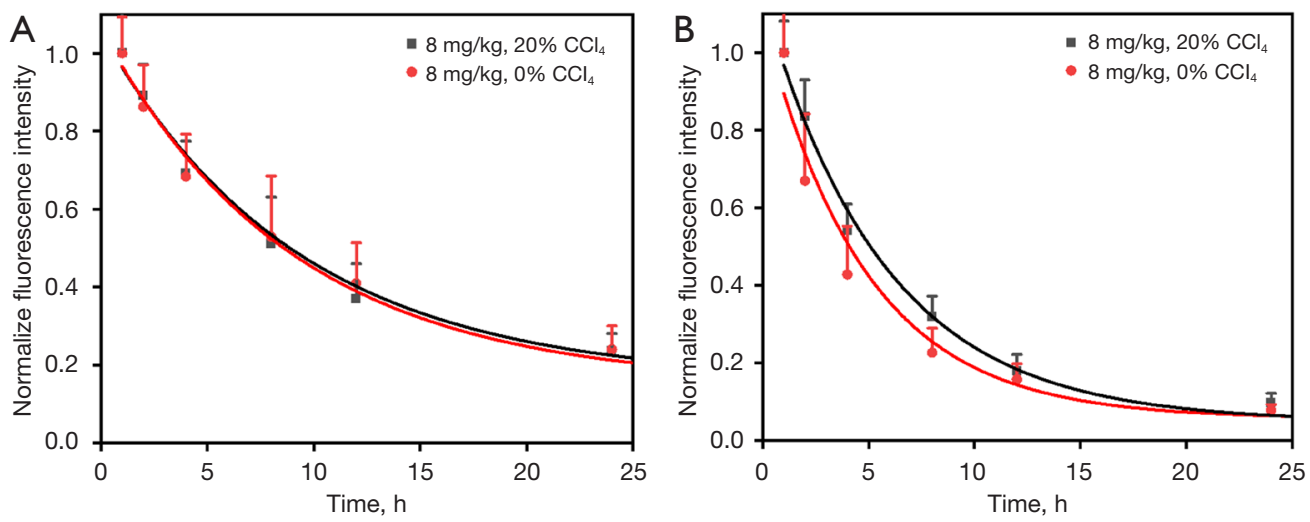

Figure 4 Normalized comparison of tumor fluorescence intensity and background fluorescence intensity for different degrees of liver injury at high dose. (A) Comparison of normalized fluorescence intensities at different time points in tumors injected with $8 \mathrm{mg} / \mathrm{kg}, 20 \% \mathrm{CCl}$ and $8 \mathrm{mg} / \mathrm{kg}, 0 \% \mathrm{CCl}_{4}$. Liver injury did not affect the rate of fluorescence intensity decay in tumors. (B) Comparison of normalized fluorescence intensities at different time points in the background of the $8 \mathrm{mg} / \mathrm{kg}, 20 \% \mathrm{CCl}_{4}$ and $8 \mathrm{mg} / \mathrm{kg}, 0 \% \mathrm{CCl}_{4}$ dose groups. Liver injury decreases the rate of fluorescence intensity decay of the background.

AST, ALP, and TBA liver function indicators in the liver injury model control group, demonstrating that $\mathrm{CCl}_{4}$ has the ability to damage liver function (Figure 5). We correlated all 8 liver function indicators with $\mathrm{I}_{0-\mathrm{T}}, \mathrm{I}_{0-\mathrm{B}}$, and $\mathrm{t}_{\text {opt }}$ one by one to obtain Pearson correlation coefficients and $\mathrm{P}$ values for each indicator with $\mathrm{I}_{0-\mathrm{T}}, \mathrm{I}_{0-\mathrm{B}}$, and $\mathrm{t}_{\mathrm{opt}}$, respectively (Table 2).

\section{Discussion}

\section{The bepatic clearance of ICG}

After intravenous injection, ICG binds to ALB in the blood to form 4-6 nm nanoparticles which accumulate inside the tumor tissue through the EPR effect (9,20,26-28). Additionally, lymphatic reflux inside the tumor tissue is blocked, while ICG in the blood is selectively absorbed by hepatocytes and then excreted unchanged into the bile through an ATP-dependent transport system (29). Our previous study showed that higher dose results in a better imaging effect (30), and when the injection dose reached $8 \mathrm{mg} / \mathrm{kg}$, the accumulation of ICG in the tumor basically reached saturation, but the TBR was the highest. The dose $(\geq 8 \mathrm{mg} / \mathrm{kg}$ ) far exceeded the average hepatic saturation dose, and TBR decreased 
Table 1 Absolute fluorescence intensity and normalized values of tumor and background at different time points

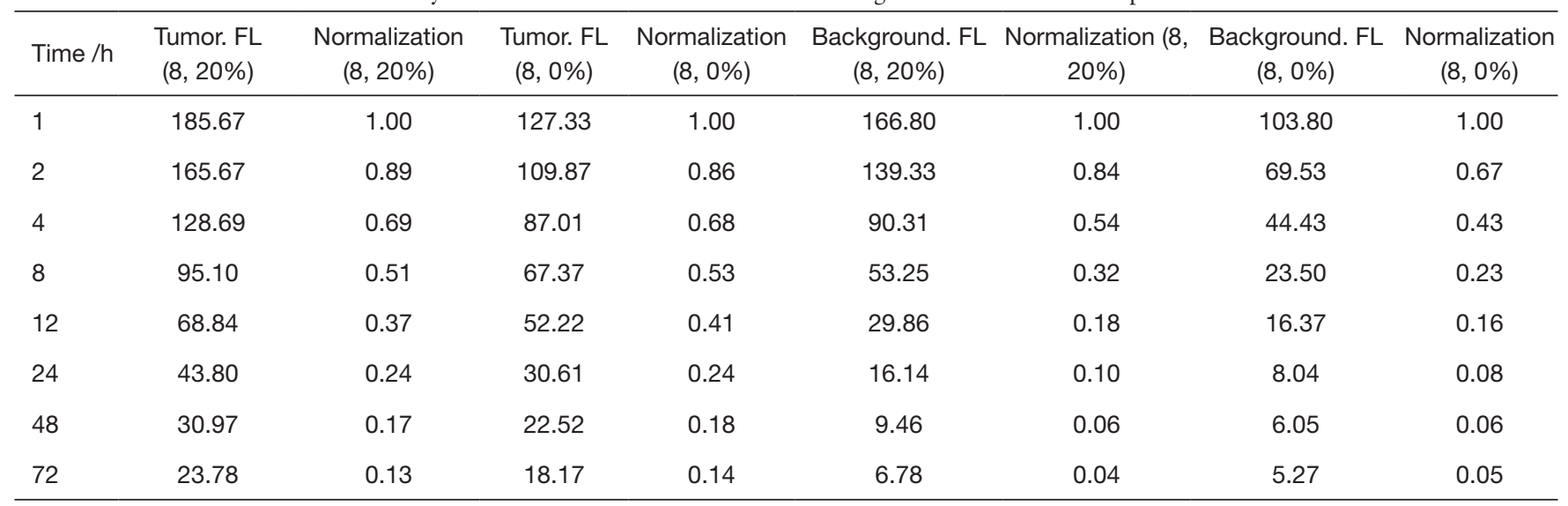

FL, fluorescence intensity; FL, fluorescence intensity; $8,20 \%$ means $8 \mathrm{mg} / \mathrm{kg}, 20 \% \mathrm{CCl}_{4} ; 8,0 \% \mathrm{means} 8 \mathrm{mg} / \mathrm{kg}, 0 \% \mathrm{CCl}_{4}$.

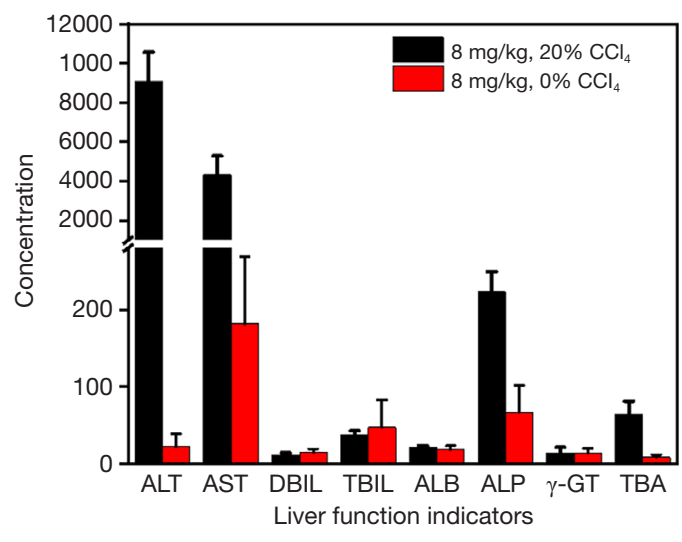

Figure 5 Liver function indicators were measured in the $8 \mathrm{mg} / \mathrm{kg}, 20 \% \mathrm{CCl}_{4}$ and $8 \mathrm{mg} / \mathrm{kg}, 0 \% \mathrm{CCl}_{4}$ dose groups. ALT, alanine aminotransferase; AST, aspartate aminotransferase; DBIL, direct bilirubin; TBIL, total bilirubin; ALB, albumin; ALP, alkaline phosphatase; $\gamma$-GT, $\gamma$-glutamyltransferase; TBA, and total bile acid.

due to slow metabolism in normal tissues. In this study, our main objective was to assess the effect of liver injury on the injected dose and optimal TBR time. The $8 \mathrm{mg} / \mathrm{kg}$ ICG group had better compositional imaging and clearer tumor borders than the $1 \mathrm{mg} / \mathrm{kg}$ group. Injection of $1 \mathrm{mg} / \mathrm{kg}$ in mice when liver function was impaired still could not effectively distinguish the tumor boundary. We suspect that the low dose of ICG did not reach its saturated metabolic range even if liver function was impaired when the low dose was injected. We obtained good imaging results after damaging the liver function of mice with $20 \%$ $\mathrm{CCl}_{4}$, and the absolute fluorescence intensity of the tumor was increased, while the tumor boundary was clearer (TBR $=3.807 \pm 0.0430$ ). At the $8 \mathrm{mg} / \mathrm{kg}$ dose, the optimal TBR time point of mice in the liver injury model group was at $72 \mathrm{~h}$, while the optimal TBR of mice in the normal liver function group appeared at $24 \mathrm{~h}$, and this can be explained based on Figure 4. We observed that liver function impairment was associated with ICG accumulation in the liver and an enhancement in ICG tumor fluorescence intensities, which was associated with impaired liver function reducing the metabolic rate of ICG. While ICG circulating time did not seem to be affected by liver injury, ICG tumor retention was basically the same for all groups. This affirms our hypothesis that the optimal TBR time is prolonged in the case of liver injury. Further optimisation experiments covering wider time points and dose ranges are needed in order to obtain a standardized protocol.

\section{Advantages of NIRFGS}

Before NIR imaging is used for biological imaging, there have been a variety of rich imaging means for research and clinical use, including MRI, CT and nuclear tomography. These existing imaging methods cannot be used for intraoperative real-time navigation due to factors such as bulky equipment, less resolution than optical imaging, expensive tracers, detection sensitivity, or radiation damage to the human body. NIR fluorescence, on the other hand, can effectively avoid the autofluorescence of biological tissues, thereby increasing the signal-to-background ratio. Because of the advantages of large penetration depth, high signal-to-background ratio and high biosafety, 
Table 2 Correlation analysis between $\mathrm{I}_{0-\mathrm{T}}, \mathrm{I}_{0-\mathrm{B}}$ and $\mathrm{t}_{\mathrm{opt}}$ and eight liver function indicators

\begin{tabular}{|c|c|c|c|c|c|c|c|c|}
\hline Parameters & ALT & AST & DBIL & TBIL & ALB & ALP & $\gamma$-GT & TBA \\
\hline $\mathrm{I}_{0-\mathrm{T}}$ & $r=0.962$ & $r=0.953$ & $r=-0.889$ & $r=-0.178$ & $r=-0.213$ & $r=0.865$ & $r=0.380$ & $r=0.945$ \\
\hline \multirow[t]{2}{*}{$\mathrm{I}_{0-B}$} & $P=0.006$ & $P=0.013$ & $P=0.008$ & $P=0.440$ & $P=0.338$ & $P=0.000$ & $P=0.243$ & $P=0.010$ \\
\hline & $r=0.912$ & $r=0.864$ & $r=-0.894$ & $r=0.081$ & $r=-0.220$ & $r=0.985$ & $r=0.358$ & $r=0.884$ \\
\hline$t_{\mathrm{opt}}$ & $r=0.894$ & $r=0.866$ & $r=-0.796$ & $\mathrm{r}=-0.282$ & $r=-0.172$ & $r=0.825$ & $r=0.600$ & $r=0.884$ \\
\hline
\end{tabular}

$P$ is significance, $r$ is the Pearson correlation coefficient, $t_{\text {opt }}$ is $t$ (TBR optimal). TBR, tumor to background ratio; ALT, alanine aminotransferase; AST, aspartate aminotransferase; DBIL, direct bilirubin; TBIL, total bilirubin; ALB, albumin; ALP, alkaline phosphatase; $\gamma$-GT, $\gamma$-glutamyltransferase; TBA, and total bile acid.

NIR fluorescence imaging is expected to be used for intraoperative real-time navigation (6-10).

\section{Advantages of CCl4 in establishing the liver injury model}

After subcutaneous injection of $\mathrm{CCl}_{4}$ corn oil solution with volume fraction of $40 \%, \mathrm{CCl}_{4}$ enters mice and can be metabolized by hepatocytes to produce trichloromethyl radicals and chloride radicals, which can induce oxidative stress, loss of enzyme function, peroxidation of the cell membrane, and ultimately liver cell injury $(31,32)$. $\mathrm{CCl}_{4}-$ induced acute liver injury in murine models is widely used to investigate potential therapeutic strategies due to its similarities with acute chemical liver injury in humans (33-36). Recently, Zhu et al. demonstrated that hepatic HMGB1 expression played a critical role in $\mathrm{CCl}_{4}$-induced acute liver injury (37). Chen et al. found that the most severe liver injury occurred at $24 \mathrm{~h}$ (38) and normalized in the following $48-72 \mathrm{~h}$. Therefore, liver function slowly recovers after $24 \mathrm{~h}$, and the gradual removal of ICG from the blood prolongs the optimal TBR time. Therefore, we chose to establish the liver injury model by intraperitoneal injection with $20 \% \mathrm{CCl}_{4}$ corn oil solution in mice, while the detection results of liver function parameters also confirmed the successful establishment of the liver injury model.

\section{Analysis of liver function indicators assisted individualized dosing regimen}

It has been reported that certain liver function parameters detected preoperatively are closely related to the metabolism of ICG. For example, it has been reported that the high bilirubin and bile acid levels in cases of liver injury could affect ICG metabolism in a competitive manner as they share the same class of membrane transporters $(39,40)$. Cholestasis affects the speed of ICG clearance, while $\gamma$-GT and ALP are related to cholestasis (41), so they are also related to the metabolic capacity of hepatic ICG. In addition, some clinical liver function scores (such as ChildPugh, MELD, MELD-Na, etc.) and other indicators which indirectly reflect liver function (such as platelet count, hyaluronic acid, hepatitis, etc.) have also been shown to be related to hepatic ICG excretion. These indicators can reflect the liver metabolism of ICG without ICG injection. Therefore, by using the relationship between preoperative liver function indicators and ICG metabolism, we established a regression analysis model to determine 4 preoperative liver function indicators which are significantly related to the TBR optimal time of ICG metabolism, namely ALT $(\mathrm{P}<0.01)$, AST $(\mathrm{P}<0.05)$, ALP $(\mathrm{P}<0.05)$, and TBA $(\mathrm{P}<0.05)$. The levels of AST and ALT are higher in liver cells and these factors are released into the blood when liver cells are damaged or die, reflecting the liver function situation. ICG is transported to the liver via serum ALB and excreted unchanged via the biliary tract. Therefore, bilirubin has a competitive, inhibitory relationship with ICG. This means that when TBA increases, it may inhibit the rate of ICG transport to the liver and affect the excretion of ICG. This is the first study to analyze the relationship between liver function indicators and ICG metabolism, which establishes a foundation for assisting doctors to develop individualized dosing regimens for patients according to liver function in the future, with high reliability and practicability.

\section{Limitations of this study}

In the future, we need to expand the sample size and 
also need to establish a variety of animal orthotopic tumor models. There is a need to establish a quantitative relationship between liver function and liver ICG metabolic capacity, which should be verified in clinical surgery.

\section{Conclusions}

This study reveals the relationship between liver excretion and optimal TBR time. Impairment of liver function will not significantly affect the maximum TBR, but will prolong the optimal TBR time. The specific liver injury can be judged based on preoperative liver function tests, and is especially correlated with ALP, AST, ALP, and TBA. When these 4 preoperative liver function parameters are elevated, it means that the excretion of ICG will be hindered, prolonging the optimal TBR time during surgery, and a wider and more stable surgical window will appear at the same time. This study showed that prolonged surgical start time is feasible according to preoperative liver function testing using NIR fluorescence imaging technology.

\section{Acknowledgments}

Funding: This study was supported by grants from the National Natural Science Foundation of China (grant 81901843), the State Commission of Science \& Technology of China (grant 2016YFC0104100), and the Jiangsu Province Science \& Technology Department (BE2016731 and BE2018698). YW acknowledges the "Jiangsu SpeciallyAppointed Professor" award.

\section{Footnote}

Reporting Checklist: The authors have completed the ARRIVE reporting checklist. Available at https://atm. amegroups.com/article/view/10.21037/atm-21-6975/rc

Data Sharing Statement: Available at https://atm.amegroups. com/article/view/10.21037/atm-21-6975/dss

Conflicts of Interest: All authors have completed the ICMJE uniform disclosure form (available at https://atm.amegroups. com/article/view/10.21037/atm-21-6975/coif). All authors report funding from State Commission of Science \& Technology of China (Grant 2016YFC0104100), National Natural Science Foundation of China (Grant 81901843), Jiangsu Province Science \& Technology Department (BE2016731 and BE2018698). HC is the owner and CEO of Nanjing Nuoyuan Medical Devices Co., Ltd. CB is the technical consultant of Nanjing Nuoyuan Medical Devices Co., Ltd. The authors have no other conflicts of interest to declare.

Ethical Statement: The authors are accountable for all aspects of the work in ensuring that questions related to the accuracy or integrity of any part of the work are appropriately investigated and resolved. Experiments were reviewed and approved by the Animal Ethical and Welfare Committee (AEWC) of Nanjing University (Approval No. IACUC-2105007), in compliance with Institutional Committee of Nanjing University guidelines for the care and use of animals.

Open Access Statement: This is an Open Access article distributed in accordance with the Creative Commons Attribution-NonCommercial-NoDerivs 4.0 International License (CC BY-NC-ND 4.0), which permits the noncommercial replication and distribution of the article with the strict proviso that no changes or edits are made and the original work is properly cited (including links to both the formal publication through the relevant DOI and the license). See: https://creativecommons.org/licenses/by-nc-nd/4.0/.

\section{References}

1. Statistics 2020: GLOBOCAN Estimates of Incidence and Mortality Worldwide for 36 Cancers in 185 Countries. CA Cancer J Clin 2021;71:209-49.

2. Chen Z, Zhang P, Xu Y, et al. Surgical stress and cancer progression: the twisted tango. Mol Cancer 2019;18:132.

3. Vahrmeijer AL, Hutteman M, van der Vorst JR, et al. Image-guided cancer surgery using near-infrared fluorescence. Nat Rev Clin Oncol 2013;10:507-18.

4. Moran MS, Schnitt SJ, Giuliano AE, et al. Society of Surgical Oncology-American Society for Radiation Oncology consensus guideline on margins for breastconserving surgery with whole-breast irradiation in stages I and II invasive breast cancer. J Clin Oncol 2014;32:1507-15.

5. Tewari A, Sooriakumaran P, Bloch DA, et al. Positive surgical margin and perioperative complication rates of primary surgical treatments for prostate cancer: a systematic review and meta-analysis comparing retropubic, laparoscopic, and robotic prostatectomy. Eur Urol 2012;62:1-15

6. Weissleder R. A clearer vision for in vivo imaging. Nat 
Biotechnol 2001;19:316-7.

7. Keating J, Tchou J, Okusanya O, et al. Identification of breast cancer margins using intraoperative near-infrared imaging. J Surg Oncol 2016;113:508-14.

8. Reinhart MB, Huntington CR, Blair LJ, et al. Indocyanine Green: Historical Context, Current Applications, and Future Considerations. Surg Innov 2016;23:166-75.

9. Kosaka N, Mitsunaga M, Longmire MR, et al. Near infrared fluorescence-guided real-time endoscopic detection of peritoneal ovarian cancer nodules using intravenously injected indocyanine green. Int J Cancer 2011;129:1671-7.

10. Martirosyan NL, Cavalcanti DD, Eschbacher JM, et al. Use of in vivo near-infrared laser confocal endomicroscopy with indocyanine green to detect the boundary of infiltrative tumor. J Neurosurg 2011;115:1131-8.

11. DSouza AV, Lin H, Henderson ER, et al. Review of fluorescence guided surgery systems: identification of key performance capabilities beyond indocyanine green imaging. J Biomed Opt 2016;21:80901.

12. Greish K. Enhanced permeability and retention of macromolecular drugs in solid tumors: a royal gate for targeted anticancer nanomedicines. J Drug Target 2007;15:457-64.

13. Troyan SL, Kianzad V, Gibbs-Strauss SL, et al. The FLARE intraoperative near-infrared fluorescence imaging system: a first-in-human clinical trial in breast cancer sentinel lymph node mapping. Ann Surg Oncol 2009;16:2943-52.

14. Jewell EL, Huang JJ, Abu-Rustum NR, et al. Detection of sentinel lymph nodes in minimally invasive surgery using indocyanine green and near-infrared fluorescence imaging for uterine and cervical malignancies. Gynecol Oncol 2014;133:274-7.

15. van der Vorst JR, Schaafsma BE, Hutteman M, et al. Nearinfrared fluorescence-guided resection of colorectal liver metastases. Cancer 2013;119:3411-8.

16. Ishizawa T, Zuker NB, Kokudo N, et al. Positive and negative staining of hepatic segments by use of fluorescent imaging techniques during laparoscopic hepatectomy. Arch Surg 2012;147:393-4.

17. Keating J, Newton A, Venegas O, et al. Near-Infrared Intraoperative Molecular Imaging Can Locate Metastases to the Lung. Ann Thorac Surg 2017;103:390-8.

18. Okusanya OT, Holt D, Heitjan D, et al. Intraoperative near-infrared imaging can identify pulmonary nodules. Ann Thorac Surg 2014;98:1223-30.

19. Holt D, Okusanya O, Judy R, et al. Intraoperative near- infrared imaging can distinguish cancer from normal tissue but not inflammation. PLoS One 2014;9:e103342.

20. Cao Y, Wang P, Wang Z, et al. A pilot study of nearinfrared fluorescence guided surgery for primary tumor localization and lymph node mapping in colorectal cancer. Ann Transl Med 2021;9:1342.

21. Jiang JX, Keating JJ, Jesus EM, et al. Optimization of the enhanced permeability and retention effect for nearinfrared imaging of solid tumors with indocyanine green. Am J Nucl Med Mol Imaging 2015;5:390-400.

22. Kim HK, Quan YH, Choi BH, et al. Intraoperative pulmonary neoplasm identification using near-infrared fluorescence imaging. Eur J Cardiothorac Surg 2016;49:1497-502.

23. Zeh R, Sheikh S, Xia L, et al. The second window ICG technique demonstrates a broad plateau period for near infrared fluorescence tumor contrast in glioblastoma. PLoS One 2017;12:e0182034.

24. Onda N, Kimura M, Yoshida T, et al. Preferential tumor cellular uptake and retention of indocyanine green for in vivo tumor imaging. Int J Cancer 2016;139:673-82.

25. Wang J. Application of Indocyanine Green Fluorescence Navigation in Surgery. Modern Medicine 2017; 45:1852-4.

26. Mohs AM, Mancini MC, Singhal S, et al. Hand-held spectroscopic device for in vivo and intraoperative tumor detection: contrast enhancement, detection sensitivity, and tissue penetration. Anal Chem 2010;82:9058-65.

27. Madajewski B, Judy BF, Mouchli A, et al. Intraoperative near-infrared imaging of surgical wounds after tumor resections can detect residual disease. Clin Cancer Res 2012;18:5741-51.

28. Nagahara R, Onda N, Yamashita S, et al. Fluorescence tumor imaging by i.v. administered indocyanine green in a mouse model of colitis-associated colon cancer. Cancer Sci 2018;109:1638-47.

29. Faybik P, Hetz H. Plasma disappearance rate of indocyanine green in liver dysfunction. Transplant Proc 2006;38:801-2.

30. Wang Z, Ni K, Zhang X, et al. Method for Real-Time Tissue Quantification of Indocyanine Green Revealing Optimal Conditions for Near Infrared Fluorescence Guided Surgery. Anal Chem 2018;90:7922-9.

31. Yu J, Wang Y, Qian H, et al. Polyprenols from Taxus chinensis var. mairei prevent the development of $\mathrm{CCl}_{4}$-induced liver fibrosis in rats. J Ethnopharmacol 2012;142:151-60.

32. Crocenzi FA, Roma MG. Silymarin as a new 
hepatoprotective agent in experimental cholestasis: new possibilities for an ancient medication. Curr Med Chem 2006;13:1055-74.

33. Dai C, Xiao X, Li D, et al. Chloroquine ameliorates carbon tetrachloride-induced acute liver injury in mice via the concomitant inhibition of inflammation and induction of apoptosis. Cell Death Dis 2018;9:1164.

34. Shi H, Han W, Shi H, et al. Augmenter of liver regeneration protects against carbon tetrachloride-induced liver injury by promoting autophagy in mice. Oncotarget 2017;8:12637-48

35. Zhang DG, Zhang C, Wang JX, et al. Obeticholic acid protects against carbon tetrachloride-induced acute liver injury and inflammation. Toxicol Appl Pharmacol 2017;314:39-47.

36. Torres LR, Santana FC, Torres-Leal FL, et al. Pequi (Caryocar brasiliense Camb.) almond oil attenuates carbon tetrachloride-induced acute hepatic injury in rats: Antioxidant and anti-inflammatory effects. Food Chem

Cite this article as: Li Y, You Q, Wang Z, Cao Y, Butch CJ, Guissi NEI, Cai H, Wang Y, Lu Q. A study on setting standards for near-infrared fluorescence-image guided surgery (NIRFGS) time lapse monitoring based on preoperative liver function assessment. Ann Transl Med 2022;10(2):96. doi: 10.21037/atm21-6975
Toxicol 2016;97:205-16.

37. Zhu RZ, Xiang D, Xie C, et al. Protective effect of recombinant human $\mathrm{IL}-1 \mathrm{Ra}$ on $\mathrm{CCl}$-induced acute liver injury in mice. World J Gastroenterol 2010;16:2771-9.

38. Chen M, Huang W, Wang C, et al. High-mobility group box 1 exacerbates $\mathrm{CCl}_{4}$-induced acute liver injury in mice. Clin Immunol 2014;153:56-63.

39. van de Steeg E, Stránecký V, Hartmannová H, et al. Complete OATP1B1 and OATP1B3 deficiency causes human Rotor syndrome by interrupting conjugated bilirubin reuptake into the liver. J Clin Invest 2012;122:519-28.

40. Sticova E, Jirsa M. New insights in bilirubin metabolism and their clinical implications. World J Gastroenterol 2013;19:6398-407.

41. Ho RH, Tirona RG, Leake BF, et al. Drug and bile acid transporters in rosuvastatin hepatic uptake: function, expression, and pharmacogenetics. Gastroenterology 2006;130:1793-806. 

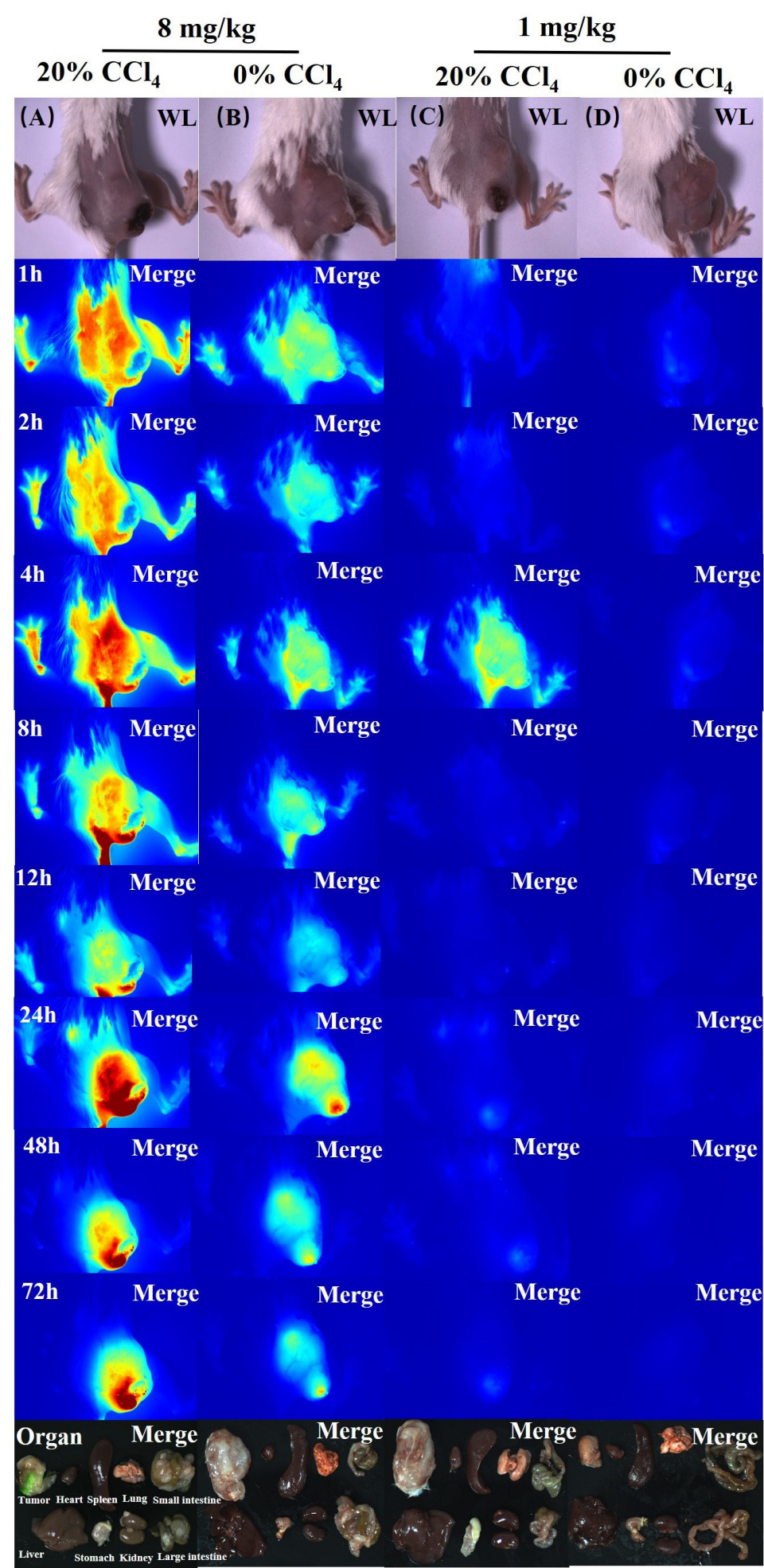

Figure S1 Tumor and organ images of mice in the imaging groups at different times. (A) Mice administered $8 \mathrm{mg} / \mathrm{kg}$ and $20 \% \mathrm{CCl} \mathrm{were}_{4}$ imaged at 1,2,4,8,12, 24, 48, and $72 \mathrm{~h}$ after injection, white light images and merged images; (B) $\mathrm{Mice}$ administered $8 \mathrm{mg} / \mathrm{kg}$ and $0 \% \mathrm{CCl}_{4}$ were imaged after injection, white light images and merged images; (C) Mice administered $1 \mathrm{mg} / \mathrm{kg}$ and $20 \% \mathrm{CCl}_{4}$ were imaged after injection, white light images and merged images; (D) Mice administered $1 \mathrm{mg} / \mathrm{kg}$ and $0 \% \mathrm{CCl}_{4}$ were imaged after injection, white light images and merged images. 